University of Nebraska - Lincoln

DigitalCommons@University of Nebraska - Lincoln

Faculty Papers and Publications in Animal

Science

Animal Science Department

January 1993

\title{
Sequential Transformation for Multiple Traits for Estimation of (Co)variance Components with a Derivative-Free Algorithm for Restricted Maximum Likelihood
}

\author{
L. Dale Van Vleck \\ University of Nebraska-Lincoln, dvan-vleck1@unl.edu \\ K. G. Boldman \\ DeKalb Poultry Research, Inc.
}

Follow this and additional works at: https://digitalcommons.unl.edu/animalscifacpub

Part of the Animal Sciences Commons

Van Vleck, L. Dale and Boldman, K. G., "Sequential Transformation for Multiple Traits for Estimation of (Co)variance Components with a Derivative-Free Algorithm for Restricted Maximum Likelihood" (1993). Faculty Papers and Publications in Animal Science. 253.

https://digitalcommons.unl.edu/animalscifacpub/253

This Article is brought to you for free and open access by the Animal Science Department at DigitalCommons@University of Nebraska - Lincoln. It has been accepted for inclusion in Faculty Papers and Publications in Animal Science by an authorized administrator of DigitalCommons@University of Nebraska - Lincoln. 


\title{
Sequential Transformation for Multiple Traits for Estimation of (Co)/variance Components with a Derivative-Free Algorithm for Restricted Maximum Likelihood ${ }^{1,2}$
}

\author{
L. D. Van Vleck and K. G. Boldman ${ }^{3}$ \\ Roman L. Hruska U.S. Meat Animal Research Center, ARS, USDA, Clay Center, NE 68933-0166 \\ and Department of Animal Science, University of Nebraska, Lincoln 68583-0908
}

\begin{abstract}
Transformation of multiple-trait records that undergo sequential selection can be used with derivative-free algorithms to maximize the restricted likelihood in estimation of covariance matrices as with derivative methods. Data transformation with appropriate parts of the Choleski decomposition of the current estimate of the residual covariance matrix results in mixed-model equations that are easily modified from round to round for calculation of the logarithm of the likelihood. The residual sum of squares is the same for transformed and untransformed analyses. Most importantly, the logarithm of the determinant of the untransformed coefficient matrix is an easily determined function of the

Choleski decomposition of the residual covariance matrix and the determinant of the transformed coefficient matrix. Thus, the logarithm of the likelihood for any combination of covariance matrices can be determined from the transformed equations. Advantages of transformation are 1) the multiple-trait mixed-model equations are easy to set up, 2 ) the least squares part of the equations does not change from round to round, 3) right-hand sides change from round to round by constant multipliers, and 4) less memory is required. An example showed only a slight advantage of the transformation compared with no transformation in terms of solution time for each round ( 1 to $5 \%$ ).
\end{abstract}

Key Words: Multiple Trait Analysis, Covariance Estimation, Transformation, Restricted Maximum Likelihood

J. Anim. Sci. 1993. 71:836-844

\section{Introduction}

Derivative methods of estimating variance components to maximize the restricted likelihood (REML; Patterson and Thompson, 1971) require elements from the inverse of the coefficient matrix of the mixedmodel equations (Dempster et al., 1977). Time for inversion provides a limit to the number of equations for derivative methods. Smith and Graser (1986) and Graser et al. (1987) introduced a derivative-free (DFREML) procedure that maximizes the likelihood

\footnotetext{
${ }^{1}$ Published as paper no. 9959, Journal Ser., Nebraska Agric. Res. Div., Univ. of Nebraska, Lincoln 68583-0908.

${ }^{2}$ The sparse-matrix package, SPARSPAK, is a licensed product from the Computer Sci. Dept., Univ. of Waterloo, ON, Canada, and the Microway NDP 386 FORTRAN compiler is a licensed product from Microway, Inc., Research Park, Box 79, Kingston, MA 02364. The Weitek 4167 coprocessor is a product of Weitek Corp., $1060 \mathrm{E}$. Arques Avenue, Sunnyvale, CA 94086.

${ }^{3}$ Current address: DeKalb Poultry Research, Inc., 3100 Sycamore Road, DeKalb, IL 60115.

Received June 22, 1992.

Accepted November 2, 1992.
}

by using a search procedure whereby the logarithm of the likelihood is calculated for combinations of estimates of parameters until the combination that maximizes the likelihood is found. Inversion of the coefficient matrix is not required. The algorithm they described required Gaussian elimination with sparsematrix methods that is much more efficient than inversion. Meyer $(1988,1989,1991)$ extended the procedure to complex models with direct and maternal genetic effects with covariance and an independent random effect (e.g., litter or permanent environment), in addition to a random residual effect.

The DFREML algorithm of Meyer (1989) minimized storage requirements with linked-list techniques to increase the efficiency of Gaussian elimination and expanded dramatically the number of equations that could be considered in estimating secondorder parameters with REML. Boldman and Van Vleck (1991) developed a different algorithm for calculating the residual sum of squares and logarithm of the determinant of the coefficient matrix of mixedmodel equations that are needed to calculate the logarithm of the likelihood. Their method was based on the use of a sparse-matrix package (e.g., SPAR- 
SPAK, George et al., 1980), to reorder the coefficient matrix once for each design pattern followed in each round by a sparse Choleski decomposition that yields rather directly the log determinant of the coefficient matrix and solutions from which the residual sum of squares can be calculated. All other steps are as described by Meyer (1989). The algorithm with the SPARSPAK routines resulted in two levels of magnitude decrease in time needed to reach convergence (100 to 600 times less computer time) compared with the original version of DFREML (Meyer, 1988). Sparse-matrix techniques increase the feasibility of derivative-free procedures for estimation of variances and covariances for multiple-trait models for cases when not all traits are measured on all animals. The canonical transformation (e.g., Thompson, 1977; Arnason, 1982; Lawlor, 1984; Quaas, 1984; Meyer, 1985) reduces the multiple-trait problem to a series of single-trait analyses if all traits are measured on all animals, the design matrices are the same for all traits, and the model contains only one random factor other than a residual effect. Pollak and Quaas (1982) proposed a transformation of the residual covariance matrix for setting up the mixed-model equations when all traits are not measured for the case where the measured traits follow a sequential pattern. This idea was expanded to estimation of variance components for a multiple-trait model by Walter et al. (1986) and Garrick (1988) for use with derivative methods of obtaining REML estimates of variances and covariances of multiple traits.

The purpose of this paper is to describe a derivative-free procedure to obtain REML estimates for traits available sequentially (i.e., an animal with a measured third trait also has measurements for the first and second traits and an animal with a measured second trait also has a measured first trait). Many multiple-trait data sets follow this pattern whereby selection on an early trait determines whether the animal will have a later trait measured. Thus, the traits on which selection are based will be included in the analysis so that the apparent property of REML to account for such selection will be used (Sorensen and Kennedy, 1983, 1984, 1986). The other requirement is that the model be the same for all traits measured on an animal. This requirement is not always restrictive; dummy fixed effects could sometimes be assigned to obtain equal design matrices in cases in which the sequential fixed factors are different for the traits at the expense of increases in sampling variances.

\section{Methods}

Harville (1977) and Searle (1979) presented the identities used by Smith and Graser (1986), Graser et al. (1987), and Meyer (1989) to calculate the logarithm of the normal likelihood. The expression applies to multivariate multiple as well as single-trait normal models. The general model for single or multiple traits is as follows:

$$
\mathbf{y}=\mathbf{X} \boldsymbol{\beta}+\mathbf{Z u}+\mathbf{e}
$$

with

$$
\begin{aligned}
\mathbf{y}= & \text { vector of observations, } \\
\beta & =\text { vector of fixed effects associated with } \mathbf{y} \\
& \text { by matrix } \mathbf{X} \\
\mathbf{u}= & \text { vector of random effects associated } \\
& \text { with } \mathbf{y} \text { by matrix } \mathbf{Z} \text {, and } \\
\mathbf{e}= & \text { vector of residual effects with } \\
\mathrm{E}[\mathbf{y}]= & \mathbf{X} \boldsymbol{\beta}, \text { and } \mathrm{E}[\mathbf{u}]=0 \text { and } \mathrm{E}[\mathbf{e}]=\mathbf{0}
\end{aligned}
$$$$
\mathbf{V}\left[\begin{array}{l}
\mathbf{y} \\
\mathbf{u} \\
\mathbf{e}
\end{array}\right]=\left[\begin{array}{ccc}
\mathbf{Z G Z ^ { \prime } + \mathbf { R }} & \mathbf{Z G} & \mathbf{R} \\
\mathbf{G} \mathbf{Z}^{\prime} & \mathbf{G} & 0 \\
\mathbf{R} & 0 & \mathbf{R}
\end{array}\right]
$$

Note $\mathbf{V}(\mathbf{y})=\mathbf{V}=\mathbf{Z G Z}+\mathbf{R}$ where, for example, $\mathbf{G}$ and $\mathbf{R}$ for $t$ traits are the genetic and environmental covariance matrices.

The untransformed mixed-model equations ( MME) for single or multiple-trait models are as follows:

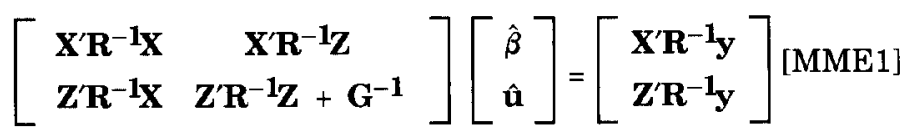

The logarithm of the multivariate normal likelihood given a sample, $\mathbf{y}$, is (e.g., Harville, 1977; Searle, 1979):

$\Lambda=-.5\left[\right.$ constant $\left.+\log |\mathbf{R}|+\log |\mathbf{G}|+\log |\mathbf{C}|+\mathbf{y}^{\prime} \mathbf{P y}\right]$.

The costly terms to compute are $\log |\mathbf{C}|$, the logarithm of the determinant of coefficient matrix for the MME, and $\mathbf{y}^{\prime} \mathbf{P y}=\mathbf{y}^{\prime} \mathbf{R}^{-\mathbf{1}} \mathbf{y}-\hat{\boldsymbol{\beta}} \mathbf{X}^{\prime} \mathbf{R}^{-\mathbf{1}} \mathbf{y}-\hat{\mathbf{u}}^{\prime} \mathbf{Z}^{\prime} \mathbf{R}^{-\mathbf{1}} \mathbf{y}$, the generalized residual sum of squares, where $\mathbf{P}=$ $\mathbf{V}^{-1}-\mathbf{V}^{-1} \mathbf{X}\left(\mathbf{X}^{\prime} \mathbf{V}^{-1} \mathbf{X}\right)^{-1} \mathbf{X}^{\prime} \mathbf{V}^{-1}$ and $\mathbf{V}^{-1}=\left(\mathbf{Z G} \mathbf{Z}^{\prime}+\mathbf{R}\right)^{-1}=$ $\mathbf{R}^{-1}-\mathbf{R}^{-\mathbf{1}} \mathbf{Z}\left(\mathbf{Z}^{\prime} \mathbf{R}^{-\mathbf{1}} \mathbf{Z}+\mathbf{G}^{-\mathbf{1}}\right)^{-\mathbf{1}} \mathbf{Z}^{\prime} \mathbf{R}^{-\mathbf{1}}$ are used in showing the identity (e.g., Henderson, 1984). The other two terms do not depend on the mixed-model equations and are relatively easy to compute (e.g., Meyer, 1989, 1991).

The transformation on traits measured sequentially results in a transformed vector of observations, $\mathbf{y} *$, such that the variance of $\mathbf{e}^{*}$ is $\mathbf{R}_{*}=\mathbf{I}$. For $\mathbf{y}_{\mathbf{i}}$ the vector of observations on $t$ traits for animal $i$, the transformed vector is $\mathbf{y}_{\mathbf{i}}^{*}=\mathbf{L}_{\mathbf{i}}^{-1} \mathbf{y}_{\mathbf{i}}$ where $\mathbf{L}_{\mathbf{i}}$ is the inverse of the Choleski decomposition of $\mathbf{R}_{\mathbf{i}}$, the residual variance-covariance matrix of $\mathbf{e}_{\mathbf{i}}$. The key to this transformation (Pollak and Quaas, 1982) is that for $\mathbf{L}_{\mathbf{o}}$, the Choleski decomposition of $\mathbf{R}_{\mathbf{o}}$, the residual covariance matrix when all traits are measured, the elements of $\mathbf{L}_{\mathbf{i}}^{-\mathbf{1}}$ for fewer sequential traits than the maximum are functions of the elements of $\mathbf{L}_{\mathbf{o}}^{-1}$; for example, for one, two, and three traits, respectively, the $\mathbf{L}_{\mathbf{i}}^{-1}$ are as follows: 


$$
\left[l^{11}\right],\left[\begin{array}{cc}
l^{11} & 0 \\
l^{21} & l^{22}
\end{array}\right] \text {, and }\left[\begin{array}{ccc}
l^{11} & 0 & 0 \\
l^{21} & l^{22} & 0 \\
l^{31} & l^{32} & l^{33}
\end{array}\right] \text {. }
$$

With $\mathbf{R}_{*}=$ I, the transformed MME are as follows:

$$
\left[\begin{array}{ll}
\mathbf{X X} & \mathbf{X}^{\prime} \mathbf{Z} \\
\mathbf{Z X} & \mathbf{Z}^{\prime} \mathbf{Z}+\mathbf{G}_{*}^{-1}
\end{array}\right]\left[\begin{array}{l}
\hat{\beta}_{*} \\
\hat{\mathbf{u}}_{*}
\end{array}\right]=\left[\begin{array}{l}
\mathbf{X}^{\prime} \mathbf{y}_{*} \\
\mathbf{Z}^{\prime} \mathbf{y}^{*}
\end{array}\right]
$$

where $\mathbf{G}^{*}$ is a function of $\mathbf{G}$ and $\mathbf{L}_{\mathbf{0}}$. For example, if $\mathbf{G}$ $=\mathbf{A} \otimes \mathbf{G}_{\mathbf{0}}$, with $\otimes$ the direct product operator, $\mathbf{G}_{\mathbf{0}}$ the genetic covariance matrix, and $\mathbf{A}$ the numerator relationship matrix, then $\mathbf{G}_{*}=\mathbf{A} \otimes \mathbf{G}_{\boldsymbol{*}_{\mathrm{o}}}$ with $\mathbf{G}_{\boldsymbol{*}_{\mathrm{o}}}=$ $\mathbf{L}_{0}^{-1} \mathbf{G}_{0} \mathbf{L}_{0}^{-T}$.

The transformed equations are simple to form because $R_{*}=\mathbf{I}$ and can be easily reformed for different values of $\mathbf{R}_{\mathbf{0}}$ and $\mathbf{G}_{\mathbf{0}}$ because the only part of the coefficient matrix that changes is $\mathbf{G}_{*}^{-1}$. The updated right-hand sides are a simple function of $\mathbf{X}^{\prime} \mathbf{y}$ and $\mathbf{Z}^{\prime} \mathbf{y}$ (Pollak and Quaas, 1982; Walter et al., 1986). Can the transformed equations be used to calculate the likelihood that is to be maximized (i.e., that associated with the untransformed equations)? The answer is yes, but with some conditions. Obviously $\log |\mathbf{R}|$ and $\log |\mathbf{G}|$ can be computed in any case. But can $\mathbf{y}^{\prime} \mathbf{P y}$ and $\log |\mathbf{C}|$ be computed from $\mathbf{C}$ and $\mathbf{y} *$ ?

It can be shown that

$$
\mathbf{y}^{\prime} \mathbf{P y}=\hat{y}_{*}^{\prime} \mathbf{y}_{*}-\hat{\beta}_{*}^{\prime} \mathbf{x}_{\mathbf{y}}^{\prime}-\hat{\mathbf{u}}_{*}^{\prime} \mathbf{z}_{\mathbf{y}}^{\prime}
$$

Note, for example, that $\mathbf{y}^{\prime} \mathbf{R}^{-\mathbf{1}} \mathbf{y}=\sum_{\mathbf{i}} \mathbf{y}_{\mathbf{i}}{ }^{\prime} \mathbf{R}_{\mathbf{i}}{ }^{-\mathbf{1}} \mathbf{y}_{\mathbf{i}}$, where $\mathbf{y}_{\mathbf{i}}$ is the vector of records on animal $i$ and that $\mathbf{y}_{*}^{\prime} \mathbf{y}_{*}=$ $\sum_{\mathbf{i}} \mathbf{y}_{\mathbf{i}} \mathbf{L}_{\mathbf{i}}^{-\mathbf{T}} \mathbf{L}_{\mathbf{i}}^{-1} \mathbf{y}_{\mathbf{i}}=\Sigma \mathbf{y}_{\mathbf{i}}{ }^{\prime} \mathbf{R}_{\mathbf{i}}^{-1} \mathbf{y}_{\mathbf{i}}$

The example will show that $\log |\mathbf{C}|=\log \left|\mathbf{C}_{*}\right|+$ function of elements of $\log \left|\mathbf{L}_{\mathbf{o}}^{-1}\right|$. Thus, at every round of iteration, the same likelihood calculation can be obtained for sequentially transformed and untransformed equations. The function of $\log \left|\mathbf{L}_{\mathbf{o}}^{-1}\right|$ is a weighted function of $\log \left|\mathbf{L}_{\mathbf{1}}^{-1}\right|, \ldots, \log \left|\mathbf{L}_{\mathbf{0}}^{-1}\right|$ and numbers of different kinds of equations as will be illustrated in the example for animals measured on Trait 1 or on Traits 1 and 2 .

\section{Example With Two Traits and One Random Factor}

One complication is that levels of fixed factors associated with records of animals measured on only Trait 1 may be different from the levels of fixed factors for Trait 1 associated with records of animals measured on both Traits 1 and 2 . With more than two traits (e.g., three traits), the preceding situation extends to levels of fixed factors for Traits 1 and 2 that are different from levels of fixed factors for Traits 1 and 2 associated with animals measured on Traits 1,2 , and 3 . These unique equations determine the function of elements of $\log \left|\mathbf{L}_{\mathbf{0}}^{-1}\right|$ needed in calculation of $\log |\mathbf{C}|$.

The model for the two-trait case is as follows:

$$
\begin{gathered}
{\left[\begin{array}{l}
\mathbf{y}_{10} \\
\mathbf{y}_{11} \\
\mathbf{y}_{12} \\
\mathbf{y}_{22}
\end{array}\right]=\left[\begin{array}{lll}
\mathbf{x}_{0} & 0 & 0 \\
0 & \mathbf{x}_{1} & 0 \\
0 & \mathbf{x}_{2} & 0 \\
0 & 0 & \mathbf{x}_{2}
\end{array}\right]\left[\begin{array}{l} 
\\
\mathbf{b}_{0} \\
\mathbf{b}_{1} \\
\mathbf{b}_{2}
\end{array}\right]+} \\
{\left[\begin{array}{llll}
\mathbf{z}_{0} & 0 & 0 & 0 \\
0 & \mathbf{z}_{1} & 0 & 0 \\
0 & 0 & \mathbf{Z}_{2} & 0 \\
0 & 0 & 0 & \mathbf{Z}_{2}
\end{array}\right]\left[\begin{array}{l}
\mathbf{u}_{10} \\
\mathbf{u}_{11} \\
\mathbf{u}_{12} \\
\mathbf{u}_{22}
\end{array}\right]+\left[\begin{array}{l}
\mathbf{e}_{10} \\
\mathbf{e}_{11} \\
\mathbf{e}_{12} \\
\mathbf{e}_{22}
\end{array}\right]}
\end{gathered}
$$

where

$\mathbf{y}_{10}=$ vector of first records for animals with only records on first trait and with different levels of fixed effects from records on first trait with matching records on second trait,

$\mathbf{y}_{11}=$ vector of records on first trait for animals with only records on first trait and with levels of fixed effects in common with records on first trait with matching records on second trait,

$\mathbf{y}_{12}=$ vector of records on first trait for animals with records on both first and second traits,

$\mathbf{y}_{22}=$ vector of records on second trait corresponding to records in $\mathbf{y}_{12}$,

$\mathbf{b}_{0}=$ vector of fixed effects for Trait 1 unique to records in $\mathbf{y}_{10}$ with association matrix $\mathbf{X}_{0}$,

$\mathbf{b}_{1}=$ vector of fixed effects for Trait 1 associated with records in $\mathbf{y}_{11}$ and $\mathbf{y}_{12}$ by matrices $\mathbf{X}_{1}$ and $\mathbf{X}_{2}$,

$\mathbf{b}_{2}=$ vector of fixed effects for Trait 2 associated with records in $\mathbf{y}_{22}$ by $\mathbf{X}_{2}$ with same number of factors and levels as $\mathbf{b}_{1}$,

$\mathbf{u}_{1 j}=$ vector of additive genetic values for Trait 1 for animals in $\mathbf{y}_{10}(j=0), \mathbf{y}_{11}(j$ $=1)$, and $\mathbf{y}_{12}(j=2)$ associated with records in $\mathbf{y}_{1 \mathrm{j}}$ by matrix $\mathbf{Z}_{\mathrm{j}}$,

$\mathbf{u}_{22}=$ vector of additive genetic values for Trait 2 for animals associated with records in $\mathbf{y}_{22}$ (those with both first and second traits measured) by matrix $\mathbf{z}_{2}$

$\mathbf{u}_{20}, \mathbf{u}_{21}=$ vectors of additive genetic values for Trait 2 for animals without measures on Trait 2, 
$\mathbf{e}_{i j}=$ vector of environmental effects as$\mathrm{E}[\mathbf{y}]=\mathbf{X b}$,

$\mathbf{R}=\mathbf{V}\left[\begin{array}{l}\mathbf{e}_{10} \\ \mathbf{e}_{11} \\ \mathbf{e}_{12} \\ \mathbf{e}_{22}\end{array}\right]=\left[\begin{array}{llll}\mathbf{I}_{0} \mathrm{r}_{11} & 0 & 0 & 0 \\ 0 & \mathbf{I}_{1} \mathrm{r}_{11} & 0 & 0 \\ 0 & 0 & \mathbf{I}_{2} \mathrm{r}_{11} & \mathbf{I}_{2} \mathrm{r}_{12} \\ 0 & 0 & \mathbf{I}_{2} \mathrm{r}_{12} & \mathbf{I}_{2} \mathbf{r}_{22}\end{array}\right]$
$\mathbf{G}=\mathbf{v}\left[\begin{array}{l}\mathbf{u}_{10} \\ \mathbf{u}_{20} \\ \mathbf{u}_{11} \\ \mathbf{u}_{21} \\ \mathbf{u}_{12} \\ \mathbf{u}_{22}\end{array}\right]=\left[\begin{array}{llllll}\mathbf{g}_{11} \mathbf{A}_{00} & \mathbf{g}_{12} \mathbf{A}_{00} & \mathbf{g}_{11} \mathbf{A}_{01} & \mathbf{g}_{12} \mathbf{A}_{01} & \mathbf{g}_{11} \mathbf{A}_{02} & \mathbf{g}_{12} \mathbf{A}_{02} \\ \mathbf{g}_{12} \mathbf{A}_{00} & \mathbf{g}_{22} \mathbf{A}_{00} & \mathbf{g}_{12} \mathbf{A}_{01} & \mathbf{g}_{22} \mathbf{A}_{01} & \mathbf{g}_{12} \mathbf{A}_{02} & \mathbf{g}_{22} \mathbf{A}_{02} \\ \mathbf{g}_{11} \mathbf{A}_{10} & \mathbf{g}_{12} \mathbf{A}_{10} & \mathbf{g}_{11} \mathbf{A}_{11} & \mathbf{g}_{12} \mathbf{A}_{11} & \mathbf{g}_{11} \mathbf{A}_{12} & \mathbf{g}_{12} \mathbf{A}_{12} \\ \mathbf{g}_{12} \mathbf{A}_{10} & \mathbf{g}_{22} \mathbf{A}_{10} & \mathbf{g}_{12} \mathbf{A}_{11} & g_{22} \mathbf{A}_{11} & \mathbf{g}_{12} \mathbf{A}_{12} & \mathbf{g}_{22} \mathbf{A}_{12} \\ \mathbf{g}_{11} \mathbf{A}_{20} & \mathbf{g}_{12} \mathbf{A}_{20} & \mathbf{g}_{11} \mathbf{A}_{21} & \mathbf{g}_{12} \mathbf{A}_{21} & \mathbf{g}_{11} \mathbf{A}_{22} & \mathbf{g}_{12} \mathbf{A}_{22} \\ \mathbf{g}_{12} \mathbf{A}_{20} & \mathbf{g}_{22} \mathbf{A}_{20} & \mathbf{g}_{12} \mathbf{A}_{21} & \mathbf{g}_{22} \mathbf{A}_{21} & \mathbf{g}_{12} \mathbf{A}_{22} & \mathbf{g}_{22} \mathbf{A}_{22}\end{array}\right]$
$\mathbf{R}_{0}=\left[\begin{array}{ll}\mathbf{r}_{11} & \mathbf{r}_{12} \\ \mathbf{r}_{12} & \mathbf{r}_{22}\end{array}\right], \mathbf{G}_{\mathbf{0}}=\left[\begin{array}{ll}\mathbf{g}_{11} & \mathbf{g}_{12} \\ \mathbf{g}_{12} & \mathbf{g}_{22}\end{array}\right]$ and $\mathbf{A}=\left[\begin{array}{llll}\mathbf{A}_{00} & \mathbf{A}_{01} & \mathbf{A}_{02} \\ \mathbf{A}_{10} & \mathbf{A}_{11} & \mathbf{A}_{12} \\ \mathbf{A}_{20} & \mathbf{A}_{21} & \mathbf{A}_{22}\end{array}\right]$

is the numerator relationship matrix among animals with records in $\mathbf{y}_{10}, \mathbf{y}_{11}$, and $\mathbf{y}_{12}$.

The coefficient matrix, $\mathbf{C}$, for the untransformed mixed-model equations for equations in order $\left(b_{0} b_{1} b_{2} u_{10}\right.$ $\begin{array}{llllll}u_{20} & u_{11} & u_{21} & u_{12} & \left.u_{22}\right)^{\prime}\end{array}$ is as follows:

\begin{tabular}{|c|c|c|c|c|c|c|c|c|}
\hline \multirow[t]{6}{*}{$\mathbf{x}_{0}^{\prime} \mathbf{x}_{0} \mathbf{r}_{11}^{-1}$} & 0 & 0 & $\mathbf{x}_{0}^{\prime} \mathbf{z}_{0} \mathbf{r}_{11}^{-1}$ & 0 & 0 & 0 & 0 & 0 \\
\hline & $\mathbf{x}_{1}^{\prime} \mathbf{x}_{1} \mathbf{r}_{11}^{-1}+\mathbf{x}_{2}^{\prime} \mathbf{x}_{2} \mathbf{r}^{11}$ & $\mathbf{x}_{2}^{\prime} \mathbf{x}_{2} \mathbf{r}^{12}$ & 0 & 0 & $\mathbf{x}_{1}^{\prime} \mathbf{z}_{1} \mathbf{r}_{11}^{-1}$ & 0 & $\mathbf{x}_{2}^{\prime} \mathbf{z}_{2} \mathbf{r}^{11}$ & $\mathbf{x}_{2}^{\prime} \mathbf{z}_{2} \mathbf{r}^{12}$ \\
\hline & & $\mathbf{x}_{2}^{\prime} \mathbf{x}_{2} r^{22}$ & 0 & 0 & 0 & 0 & $\mathbf{x}_{2}^{\prime} \mathbf{z}_{2} \mathbf{r}^{12}$ & $\mathbf{x}_{2}^{\prime} \mathbf{z}_{2} \mathbf{r}^{22}$ \\
\hline & & & $\mathbf{z}_{0}^{\prime} \mathbf{z}_{0} \mathbf{r}_{11}^{-1}+\mathbf{g}^{11} \mathbf{A}^{00}$ & $\mathbf{g}^{12} \mathbf{A}^{00}$ & $\mathbf{g}^{11} \mathbf{A}^{01}$ & $\mathbf{g}^{12} \mathbf{A}^{01}$ & $\mathrm{~g}^{11} \mathrm{~A}^{02}$ & $\mathrm{~g}^{12} \mathrm{~A}^{02}$ \\
\hline & & & & $\mathrm{g}^{22} \mathrm{~A}^{00}$ & $\mathbf{g}^{12} \mathbf{A}^{01}$ & $\mathbf{g}^{22} \mathbf{A}^{01}$ & $\mathrm{~g}^{12} \mathbf{A}^{02}$ & $\mathrm{~g}^{22} \mathrm{~A}^{02}$ \\
\hline & & & & & $\mathbf{z}_{1}^{\prime} \mathbf{z}_{1} \mathbf{r}_{11}^{-1}+\mathbf{g}^{11} \mathbf{A}^{11}$ & $\mathbf{g}^{12} \mathbf{A}^{11}$ & $\mathbf{g}^{11} \mathbf{A}^{12}$ & $\mathrm{~g}^{12} \mathbf{A}^{12}$ \\
\hline \multirow[t]{3}{*}{ Symmetric } & & & & & & $\mathbf{g}^{22} \mathbf{A}^{11}$ & $\mathbf{g}^{12} \mathbf{A}^{12}$ & $\mathrm{~g}^{22} \mathrm{~A}^{12}$ \\
\hline & & & & & & & $\mathbf{z}_{2}^{\prime} \mathbf{z}_{2} \mathbf{r}^{11}+\mathrm{g}^{11} \mathbf{A}^{22}$ & $\mathbf{Z}_{2}^{\prime} \mathrm{Z}_{2} \mathbf{r}^{12}+\mathrm{g}^{12} \mathrm{~A}^{22}$ \\
\hline & & & & & & & & $\mathbf{Z}_{2}^{\prime} \mathbf{Z}_{2} \mathbf{r}^{22}+\mathrm{g}^{22} \mathbf{A}^{22}$ \\
\hline
\end{tabular}

In direct product $(\otimes)$ notation, these reduce to:

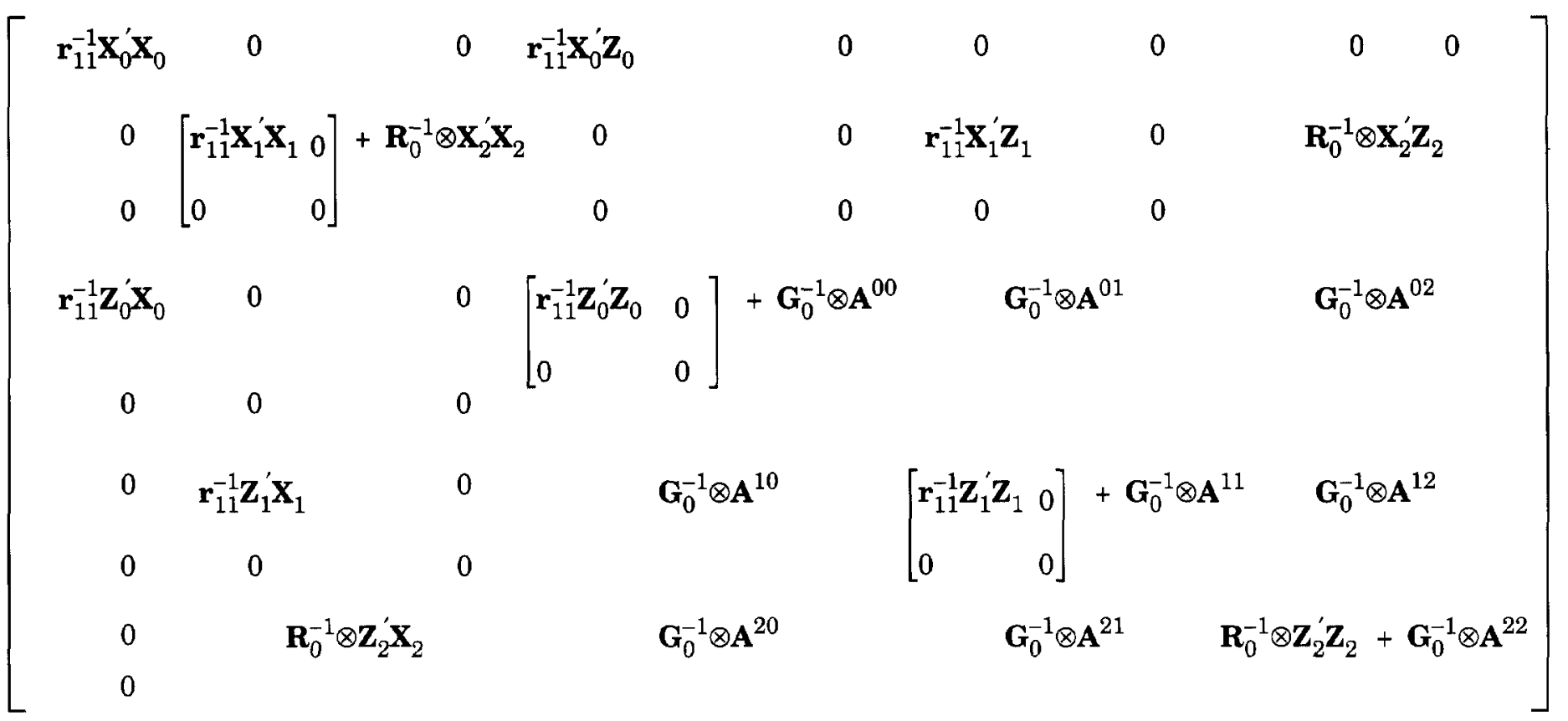


Sequential Transformation

With sequential transformation, records in $\mathbf{y}_{10}$ and $\mathbf{y}_{11}$ are multiplied by $l^{11}$ and pairs of records from $\mathbf{y}_{12}$ and $\mathbf{y}_{22}$ are multiplied by $\mathbf{L}_{0}^{-1}$, that is:

$$
\begin{gathered}
{\left[l^{11} \mathbf{I}_{0}\right] \mathbf{y}_{10}=\mathbf{y}_{10}{ }^{*},} \\
{\left[l^{11} \mathbf{I}_{1}\right] \mathbf{y}_{11}=\mathbf{y}_{11^{*}}, \text { and }} \\
{\left[\begin{array}{l}
\mathbf{L}_{0}^{-1} \mathbf{I}_{2} \\
{[}
\end{array}\right]\left[\begin{array}{l}
\mathbf{y}_{12} \\
\mathbf{y}_{22}
\end{array}\right]\left[\begin{array}{l}
l^{11} \mathbf{y}_{12} \\
l^{21} \mathbf{y}_{12}+l^{22} \mathbf{y}_{22}
\end{array}\right]=\left[\begin{array}{l}
\mathbf{y}_{12^{*}} \\
\mathbf{y}_{22^{*}}
\end{array}\right]}
\end{gathered}
$$

The coefficient matrix, $\mathbf{C} *$, for the transformed mixed-model equations is as follows:

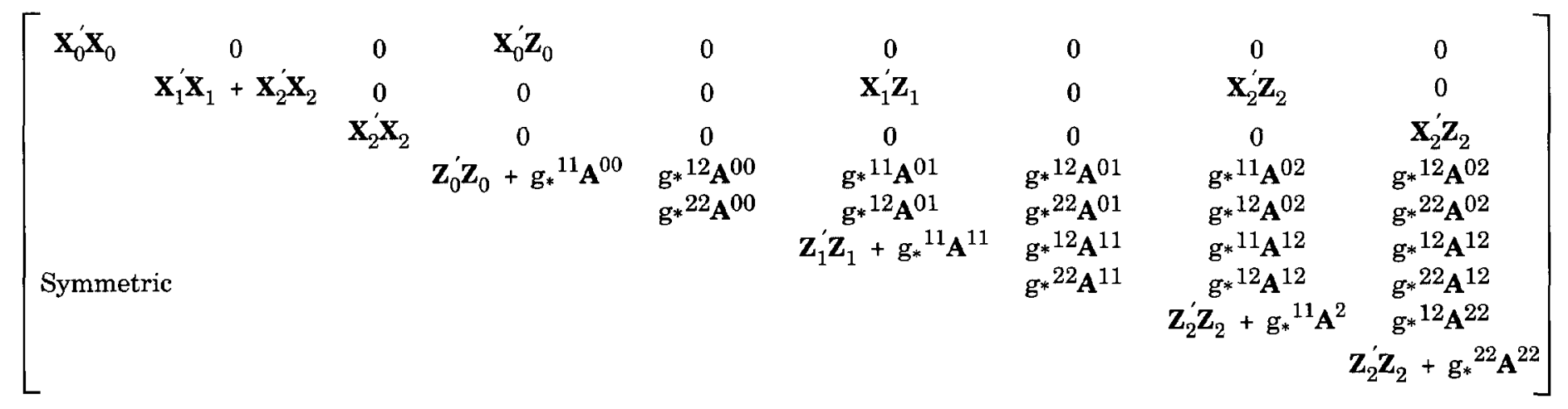

where

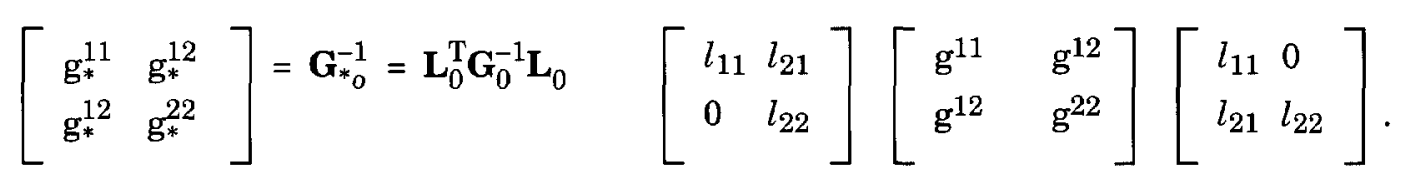

Note that

$$
\mathbf{G}_{0}^{-1}=\mathbf{L}_{0}^{-\mathrm{T}} \mathbf{G}_{*_{0}}{ }^{-1} \mathbf{L}_{0}^{-1}
$$

and from $\mathbf{L}_{0} \mathbf{L}_{0}^{\mathrm{T}}=\mathbf{R}_{\mathbf{0}}$, that $\quad\left[\begin{array}{cc}l_{11} & 0 \\ l_{21} & l_{22}\end{array}\right]\left[\begin{array}{cc}l_{11} & l_{21} \\ 0 & l_{22}\end{array}\right]=\left[\begin{array}{cc}\mathbf{r}_{11} & \mathbf{r}_{12} \\ \mathbf{r}_{12} & \mathbf{r}_{22}\end{array}\right]$;

from $\mathbf{L}_{0}^{-\mathbf{T}} \mathbf{L}_{0}^{-1}=\mathbf{R}_{\mathrm{o}}^{-1}$ that $\quad\left[\begin{array}{cc}l^{11} l^{11}+l^{21} l^{21} & l^{21} l^{22} \\ l^{21} l^{22} & l^{22} l^{22}\end{array}\right]=\left[\begin{array}{cc}\mathrm{r}^{11} & \mathrm{r}^{12} \\ \mathrm{r}^{12} & \mathrm{r}^{22}\end{array}\right]$; and

from $l_{11} l_{11}=\mathrm{r}_{11}$ that $l^{11} l^{11}=\mathrm{r}_{11}^{-1}$ 
SEQUENTIAL TRANSFORMATION WITH DFREML

Let $\mathbf{T}=\left[\begin{array}{cccc}l^{11} \mathbf{I}_{\mathrm{p}_{0}} & 0 & 0 & 0 \\ 0 & l^{11} \mathbf{I}_{\mathrm{p}_{2}} & l^{21} \mathbf{I}_{\mathrm{p}_{2}} & 0 \\ 0 & 0 & l^{22} \mathbf{I}_{\mathrm{p}_{2}} & 0 \\ 0 & 0 & 0 & l^{11} \mathbf{I}_{\mathrm{q}_{0}} \\ 0 & 0 & 0 & 0 \\ 0 & 0 & 0 & 0 \\ 0 & 0 & 0 & 0 \\ 0 & 0 & 0 & 0 \\ 0 & 0 & 0 & 0\end{array}\right.$

where

$$
\begin{aligned}
\mathrm{p}_{0}= & \text { number of effects in } \mathbf{b}_{0}, \\
\mathrm{p}_{2}= & \text { number of effects in } \mathbf{b}_{1} \text { and also in } \mathbf{b}_{2}, \\
\mathrm{q}_{0}= & \text { number of animals included in } \mathbf{u}_{10}, \\
\mathrm{q}_{1}= & \text { number of animals included in } \mathbf{u}_{11}, \\
\mathrm{q}_{2}= & \text { number of animals included in } \mathbf{u}_{12} \text { and also } \\
& \text { in } \mathbf{u}_{22}, \text { and } \\
\mathrm{q}= & \mathrm{q}_{\mathrm{o}}+\mathrm{q}_{1}+\mathrm{q}_{2} .
\end{aligned}
$$

The previous identities can be used to show that $\mathbf{T C} * \mathbf{T}^{\prime}=\mathbf{C}$. Therefore, if $\mathbf{C}$ is full rank, $\log |\mathbf{T}|+$ $\log \left|\mathbf{T}^{\prime}\right|+\log \left|\mathbf{C}_{*}\right|=\log |\mathbf{C}|$. Also note because of the triangular form of $\mathbf{T}$ that $\log |\mathbf{T}|=\log \left|\mathbf{T}^{\prime}\right|$. By examination of the example:

$$
\log |\mathbf{T}|=p_{o} \log \left(l^{11}\right)+\left(p_{2}+q\right) \log \left|\mathbf{L}_{o}^{-\mathbf{T}}\right|
$$

where

$$
\log \left|\mathbf{L}_{\mathbf{0}}^{-\mathrm{T}}\right|=\log \left(l^{11}\right)+\log \left(l^{22}\right) \text { for } \mathbf{R}_{\mathrm{o}} \text { of order two. }
$$

Thus

$$
\log |\mathbf{C}|=\log \left|\mathbf{C}_{*}\right|+2\left[\mathrm{p}_{0} \log \left(l^{11}\right)+\left(\mathrm{p}_{2}+\mathrm{q}\right) \log \left|\mathbf{L}_{0}^{-\mathrm{T}}\right|\right]
$$

Note that in this case:

$p_{0}$ is the number of levels of fixed factors unique to $\mathbf{y}_{10}$,

$\mathrm{p}_{2}$ is the number of levels of fixed factors unique to $\mathbf{y}_{12}$ or $\mathbf{y}_{22}$, and

$q=q_{o}+q_{1}+q_{2}$ is the number of animals in $\mathbf{u}_{1}$ and $\mathbf{u}_{2}$.

In general, $q$ will be the number of equations in $\mathbf{u}$ associated with any trait as $\log \left|\mathbf{L}_{o}^{-T}\right|$ will account for the number of traits.

If MME equations are augmented (Henderson, 1977) for base animals needed for relationships and

$\left.\begin{array}{ccccc}0 & 0 & 0 & 0 & 0 \\ 0 & 0 & 0 & 0 & 0 \\ 0 & 0 & 0 & 0 & 0 \\ l^{21} \mathbf{I}_{\mathrm{q}_{0}} & 0 & 0 & 0 & 0 \\ l^{22} \mathbf{I}_{\mathrm{q}_{0}} & 0 & 0 & 0 & 0 \\ 0 & l^{11} \mathbf{I}_{\mathrm{q}_{1}} & l^{21} \mathbf{I}_{\mathrm{q}_{1}} & 0 & 0 \\ 0 & 0 & l^{22} \mathbf{I}_{\mathrm{q}_{1}} & 0 & 0 \\ 0 & 0 & 0 & l^{11} \mathbf{I}_{\mathrm{q}_{2}} & l^{21} \mathbf{I}_{\mathrm{q}_{2}} \\ 0 & 0 & 0 & 0 & l^{22} \mathbf{I}_{\mathrm{q}_{2}}\end{array}\right]$

for animals without records (i.e., with equations for $\mathbf{u}_{1 \mathrm{~b}}$ and $\mathbf{u}_{2 \mathrm{~b}}$ ), then,

$\mathbf{T}$ will include an upper triangular block, $\mathbf{L}_{\mathbf{o}}^{-\mathrm{T}} \otimes \mathbf{I}_{\mathrm{q}_{\mathrm{b}}}$, and

$\mathbf{C}$ and $\mathbf{C} *$ will have added equations $\mathbf{u}_{1 \mathrm{~b}}$ and $\mathbf{u}_{2 \mathrm{~b}}$ tied to other $\mathbf{u}_{1 \mathrm{j}}$ and $\mathbf{u}_{2 \mathrm{j}}$ equations by $\mathbf{G}_{0}^{-1} \otimes \mathbf{A}_{+}^{-1}$ and $\mathbf{G}_{*_{0}}^{-1} \otimes \mathbf{A}_{+}^{-1}$, where (Henderson, 1976)

$$
\mathbf{A}_{+}^{-1}=\left[\begin{array}{llll}
\mathbf{A}^{00} & \mathbf{A}^{01} & \mathbf{A}^{02} & \mathbf{A}^{0 \mathrm{~b}} \\
\mathbf{A}^{10} & \mathbf{A}^{11} & \mathbf{A}^{12} & \mathbf{A}^{1 \mathrm{~b}} \\
\mathbf{A}^{20} & \mathbf{A}^{21} & \mathbf{A}^{22} & \mathbf{A}^{2 \mathrm{~b}} \\
\mathbf{A}^{\mathrm{b} 0} & \mathbf{A}^{\mathrm{b} 1} & \mathbf{A}^{\mathrm{b} 2} & \mathbf{A}^{\mathrm{bb}}
\end{array}\right]
$$

Note that $q$ will now include the number of such animals without records as well as animals with at least one trait measured.

\section{Constraints}

Constraints may need to be imposed before decomposition of $\mathbf{C} *$. For example, the Choleski decomposition in the solve routine of SPARSPAK-A (Chu et al., 1984) requires that the equations be of full rank.

The easiest procedure is to eliminate rows and columns of $\mathbf{X}_{0}^{\prime} \mathbf{X}_{0}$ and $\mathbf{X}_{0}^{\prime} \mathbf{Z}_{0}$ to achieve a full rank set, $p_{0}^{*}$, for the $\mathbf{b}_{0}$ equations and to eliminate pairs (for the two trait analysis) of rows and columns of $\mathbf{X}_{1}^{\prime} \mathbf{x}_{1}+$ $\mathbf{x}_{2}^{\prime} \mathbf{x}_{2}, \mathbf{x}_{1}^{\prime} \mathbf{z}_{1}, \mathbf{x}_{2}^{\prime} \mathbf{z}_{2}, \mathbf{z}_{1}^{\prime} \mathbf{x}_{1}, \mathbf{z}_{2}^{\prime} \mathbf{x}_{2}, \mathbf{x}_{2}^{\prime} \mathbf{x}_{2}$ to obtain a full rank set, $\mathbf{p}_{2}^{*}$, for each trait for the $\mathbf{b}_{1}$ and $\mathbf{b}_{2}$ equations; that is, the constraints are imposed on the same levels of $\mathbf{b}_{1}$ and $\mathbf{b}_{2}$. With $\mathbf{C} *$ and $\mathbf{C}$ constrained equivalently to full rank by zeroing rows and columns, the $\mathbf{T C} * \mathbf{T}^{\prime}=$ C relationship holds but with fewer equations and fewer rows of $\mathbf{T}$. Then with $q$ different elements of $\mathbf{u}$ for each trait:

$$
\log |\mathbf{C}|=\log \left|\mathbf{C}_{\boldsymbol{*}}\right|+2\left[\mathrm{p}_{\mathrm{o}}^{*} \log \left(l^{11}\right)+\left(\mathbf{p}_{2}^{*}+\mathbf{q}\right) \log \left|\mathbf{L}_{\mathrm{o}}^{-\mathrm{T}}\right|\right]
$$




\section{A Computing Strategy with SPARSPAK for Example of Two Traits}

1. a) Compute and save sums of squares and products:

$$
\mathbf{y}_{10}^{\prime} \mathbf{y}_{10}, \mathbf{y}_{11}^{\prime} \mathbf{y}_{11}, \mathbf{y}_{12}^{\prime} \mathbf{y}_{12}, \mathbf{y}_{12}^{\prime} \mathbf{y}_{22}, \mathbf{y}_{22}^{\prime} \mathbf{y}_{22}
$$

b) Compute and save the non-zero least squares coefficients of $\mathbf{C} *$ after constraints are imposed:

$\mathbf{x}_{\mathrm{o}}^{\prime} \mathbf{x}_{\mathrm{o}}, \mathbf{x}_{\mathbf{0}}^{\prime} \mathbf{z}_{\mathbf{0}}, \mathbf{x}_{1}^{\prime} \mathbf{x}_{1}+\mathbf{x}_{2}^{\prime} \mathbf{x}_{2}, \mathbf{x}_{1}^{\prime} \mathbf{z}_{1}$,

$\mathbf{x}_{2}^{\prime} \mathbf{Z}_{2}, \mathbf{x}_{2}^{\prime} \mathbf{x}_{2}, \mathbf{x}_{2}^{\prime} \mathbf{Z}_{2}, \mathbf{z}_{\mathbf{o}}^{\prime} \mathbf{Z}_{\mathbf{o}}, \mathbf{z}_{1}^{\prime} \mathbf{z}_{1}, \mathbf{Z}_{2}^{\prime} \mathbf{Z}_{2}$

c) Compute and save after constraints are imposed:

$\mathbf{x}_{0}^{\prime} \mathbf{y}_{10}, \quad \mathbf{x}_{1}^{\prime} \mathbf{y}_{11}, \quad \mathbf{x}_{2}^{\prime} \mathbf{y}_{12}, \quad \mathbf{x}_{2}^{\prime} \mathbf{y}_{22}$ and also $\mathbf{z}_{\mathrm{o}}^{\prime} \mathbf{y}_{10}$, $\mathbf{z}_{1}^{\prime} \mathbf{y}_{11}, \mathbf{z}_{2}^{\prime} \mathbf{y}_{12}, \mathbf{z}_{2}^{\prime} \mathbf{y}_{22}$

d) Compute $\mathbf{A}_{+}^{-1}$ and if desired, $\log \left|\mathbf{A}_{+}\right|$, an unneeded constant as $4 \Sigma \log \left(\mathrm{v}_{\mathrm{i}}\right)$ with the $\mathrm{v}_{\mathrm{i}}$ used in calculation of $\mathbf{A}_{+}^{-1}$ as described by Quaas (1976).

2. Enter initial values of $\mathbf{R}_{\mathbf{0}}, \mathbf{G}_{0}$

a) Find $\mathbf{L}_{0}$ such that $\mathbf{L}_{0} \mathbf{L}_{0}^{\mathrm{T}}=\mathbf{R}_{\mathbf{o}}$ and also elements of $\mathbf{L}_{0}^{-1}, l^{11}, l^{21}, l^{22}$.

b) Compute $\mathbf{L}_{\mathrm{o}}^{\mathrm{T}} \mathbf{G}_{\mathrm{o}}^{-1} \mathbf{L}_{\mathrm{o}}$ and add $\left(\mathbf{L}_{\mathrm{o}}^{\mathrm{T}} \mathbf{G}_{\mathrm{o}}^{-1} \mathbf{L}_{\mathrm{o}}\right) \otimes \mathbf{A}_{+}^{-1}$ to least squares form of $\mathbf{C}$.

c) Compute transformed right-hand sides

$l^{11}\left(\mathbf{x}_{0}^{\prime} \mathbf{y}_{10}\right), l^{11}\left(\mathbf{x}_{1}^{\prime} \mathbf{y}_{11}+\mathbf{x}_{2}^{\prime} \mathbf{y}_{12}\right)$,

$l^{21}\left(\mathbf{X}_{2}^{\prime} \mathbf{y}_{12}\right)+l^{22}\left(\mathbf{X}_{2}^{\prime} \mathbf{y}_{22}\right)$,

$l^{11}\left(\mathbf{Z}_{\mathbf{0}}^{\prime} \mathbf{y}_{10}\right), l^{11}\left(\mathbf{Z}_{1}^{\prime} \mathbf{y}_{11}\right), l^{11}\left(\mathbf{Z}_{2}^{\prime} \mathbf{y}_{12}\right)$,

$l^{21}\left(\mathbf{Z}_{2}^{\prime} \mathbf{y}_{12}\right)+l^{22}\left(\mathbf{Z}_{2}^{\prime} \mathbf{y}_{22}\right)$

d) If equations have not been reordered (i.e., first round with that data structure), reorder equations with SPARSPAK and save reordering.

3. a) Calculate $\log |\mathbf{C} *|$ from sparse Choleski decomposition of reordered $\mathbf{C} *$ as twice the sum of logarithms of diagonal elements.

b) Solve for $\hat{\boldsymbol{\beta}}_{*}, \hat{\mathbf{u}}_{*}$ from Choleski decomposition and transformed RHS with SPARSPAK and compute REDN $=\hat{\beta} * \mathbf{X}^{\prime} \mathbf{y}^{*}+\hat{\mathbf{u}} *^{\prime} \mathbf{Z}^{\prime} \mathbf{y} *$

c) Calculate $\mathbf{y}^{\prime} \mathbf{y}^{*}$ as

$l^{11} l^{11}\left(\mathbf{y}_{10}^{\prime} \mathbf{y}_{10}+\mathbf{y}_{11}^{\prime} \mathbf{y}_{11}+\mathbf{y}_{12}^{\prime} \mathbf{y}_{12}\right)+l^{21} l^{21}\left(\mathbf{y}_{12}^{\prime} \mathbf{y}_{12}\right)$

$+2 l^{21} l^{22}\left(\mathbf{y}_{12}^{\prime} \mathbf{y}_{22}\right)+l^{22} l^{22}\left(\mathbf{y}_{22}^{\prime} \mathbf{y}_{22}\right)$

d) Calculate $\mathbf{y}^{\prime} \mathbf{P y}=\mathbf{y} * \mathbf{y} *-\operatorname{REDN}$

e) Calculate $\log |\mathbf{G}|=n_{g} \log \left|\mathbf{A}_{+}\right|+q \log \left|\mathbf{G}_{0}\right|$ where $n_{g}=2$ is number of traits (order of $G_{0}$ ) and $q$ is number of animals represented in $\mathbf{u}$. For $\mathbf{G}_{0}$ of order greater than two or with maternal variances and covariances included, an easy way to calculate $\log \left|\mathbf{G}_{0}\right|$ would be to find the Choleski factor, $\mathbf{L}_{\mathrm{g}}$, of $\mathbf{G}_{\mathbf{0}}$, then calculate $\log \left|\mathbf{G}_{0}\right|$ as twice the sum of logarithms of diagonal elements of $\mathbf{L}_{\mathrm{g}}$. f) Calculate $\log |\mathbf{R}|=\mathrm{n}_{1} \log \left(l_{11}\right)+\mathrm{n}_{2}\left[\log \left(l_{11}\right)+\right.$ $\left.\log \left(l_{22}\right)\right]$

where $n_{1}$ is number of animals with Trait 1 only and $\mathrm{n}_{2}$ is number with both Traits 1 and 2 .

g) Calculate $\log |\mathbf{C}|=\log |\mathbf{C} *|+$ $2\left[\mathrm{p}_{\mathrm{o}}^{*} \log \left(l^{11}\right)+\left\{\mathrm{p}_{2}^{*}+\mathrm{q}\right\}\left\{\log \left(l^{11}\right)+\log \left(l^{22}\right)\right\}\right]$ where $p_{o}^{*}$ is rank of $\mathbf{X}_{0}^{\prime} \mathbf{x}_{0}, p_{2}^{*}$ is number of equations after constraints for $\mathbf{b}_{1}$ or $\mathbf{b}_{2}$, and $q=q_{0}+$ $q_{1}+q_{2}+q_{b}$ is number of animals represented in u.

h) Calculate $\boldsymbol{\Lambda}=-.5[\log |\mathbf{C}|+\mathbf{y} \mathbf{P y}+\log |\mathbf{R}|+$ $\log |\mathbf{G}|]$

4. a) Use $\Lambda$ with $\mathbf{R}_{0}, \mathbf{G}_{0}$ and previous $\Lambda$ 's with associated $\mathbf{R}_{o}$ and $\mathbf{G}_{0}$ in search strategy (e.g., Simplex) to update $\mathbf{R}_{0}$ and $\mathbf{G}_{0}$,

b) Check for convergence and, if not satisfied,

c) Find updated $\mathbf{L}_{0}$ (as in $2 \mathbf{a}$ ),

d) Update and replace $\mathbf{G}_{*}^{-1}=\left(\mathbf{L}_{\mathbf{0}}^{\mathrm{T}} \mathbf{G}_{0}^{-1} \mathbf{L}_{\mathrm{o}}\right) \otimes \mathbf{A}_{+}^{-1}$ in $\mathbf{C}_{*}$ (as in 2b),

e) Update RHS with updated, $l^{11}, l^{21}, l^{22}$ (as in $2 \mathrm{c}$,

f) Enter updated equations and right-hand sides, and

g) Go to 3a) and repeat until satisfied with convergence.

\section{Comparison of Efficiency of Calculation of $\log |\mathrm{C}|$ for Transformed and Untransformed Equations}

Compared to the untransformed equations in [MME1], programming to form the transformed equations in [MME2] in each round of iteration is easier because $\mathbf{X} \mathbf{X}, \mathbf{X}^{\prime} \mathbf{Z}$ (and $\mathbf{Z}^{\prime} \mathbf{X}$ ) and $\mathbf{Z}^{\prime} \mathbf{Z}$ do not change for different values of $\mathbf{R}_{0}$ and $\mathbf{G}_{0}$. In addition, $\mathbf{X} \mathbf{X}$ and $\mathbf{X}^{\prime} \mathbf{Z}$ will have fewer non-zero elements than $\mathbf{X}^{\prime} \mathbf{R}^{-1} \mathbf{X}$ and $\mathbf{X}^{\prime} \mathbf{R}^{-1} \mathbf{Z}$ of the untransformed equations and it would seem likely that SPARSPAK would perform a different reordering, possibly resulting in faster Choleski factorization and solutions in each round.

\section{Data and Models}

A sample set of data for comparison of memory and time requirements for reordering and solution of the untransformed and transformed equations were from the Germ Plasm Utilization Project at the USDA Meat Animal Research Center (Gregory et al., 1988). Measures of three traits (birth weight, 200-d weight, and ADG) were available for 1,064 Brown Swiss calves born from 1978 to 1989 . The first multiple-trait animal model included fixed birth year and dam age $(2,3,4$, or $\geq 5$ yr) by animal sex (male or female) effects, and random animal (additive genetic) and dam (maternal permanent environmental) effects. The relationship matrix was augmented to order 1,586 
Table 1. Memory requirements and central processing unit times for reordering and solution by SPARSPAK and SPARSPAK-A of untransformed and transformed mixed-model equations for a model with and a model without

a permanent environmental effect ${ }^{a}$

\begin{tabular}{lccccc}
\hline \hline & \multicolumn{2}{c}{$\begin{array}{c}\text { Model with permanent } \\
\text { environmental effect }\end{array}$} & & & \multicolumn{2}{c}{$\begin{array}{c}\text { Model without permanent } \\
\text { environmental effect }\end{array}$} \\
\cline { 2 - 3 } \cline { 5 - 6 } Item & $\begin{array}{c}\text { Untransformed } \\
\text { equations }\end{array}$ & $\begin{array}{c}\text { Transformed } \\
\text { equations }\end{array}$ & & $\begin{array}{c}\text { Untransformed } \\
\text { equations }\end{array}$ & $\begin{array}{c}\text { Transformed } \\
\text { equations }\end{array}$ \\
\hline Memory, kbytes & 1,690 & 1,670 & & 1,241 & 1,270 \\
Reordering time, s & $\{1,688\}$ & $\{1,655\}$ & & $\{1,252\}$ & $\{1,272\}$ \\
Solution time, s & 432.37 & 316.48 & & 271.44 & 230.57 \\
& $\{43.06\}$ & $\{61.57\}$ & & $\{30.05\}$ & $\{63.28\}$ \\
& 15.05 & 14.94 & & 11.81 & 11.81 \\
& $\{15.27\}$ & $\{15.05\}$ & & $\{12.69\}$ & $\{12.03\}$ \\
\hline
\end{tabular}

a Numbers within brackets are for SPARSPAK-A.

$b_{6,225}$ equations.

$\mathrm{c}_{4,815}$ equations.

by including 522 parents without records (base animals). The data set included 470 dams with maternal permanent environmental effects for each trait (all traits measured on animals with records), resulting in a full rank, mixed-model coefficient matrix with order 6,225 , and 186,669 and 123,063 nonzero elements in the untransformed, $\mathbf{C}$, and transformed, $\mathbf{C} *$, coefficient matrices, respectively. In a second model, permanent environmental effects were omitted, resulting in a full rank mixed-model coefficient matrix of order 4,815 and 128,691 and 103,737 non-zero elements in the untransformed and transformed coefficient matrices.

For each model, the untransformed equations and the transformed equations were reordered and solved via SPARSPAK. Two versions of SPARSPAK were used: the original version of SPARSPAK (George et al., 1980) in which reordering was by a symmetric implementation of the minimum degree algorithm and SPARSPAK-A (Chu et al., 1984), in which the original minimum degree algorithm has been modified to incorporate the ideas of multiple elimination and minimum external degrees (Liu, 1983).

According to George and $\mathrm{Ng}$ (1984), the modified algorithm is faster than the previous one and also the amount of fill-in is usually smaller. Computations for each of the eight analyses (untransformed and transformed equations, model with and model without maternal permanent environmental effects, and SPARSPAK and SPARSPAK-A) were run on a 486-33 personal computer with a Weitek 4167 coprocessor using a Microway NDP 386 FORTRAN compiler.

\section{Results}

Compared with the untransformed equations, the transformed equations contained 34 and $19 \%$ fewer non-zero elements for the model with and for the model without permanent environmental effects, respectively.

Computational requirements for reordering and solution of the untransformed and transformed multiple-trait coefficient matrices by original SPARSPAK and SPARSPAK-A for the two models are shown in Table 1. For original SPARSPAK, the time required for reordering in each model was reduced after transformation with a greater reduction for the full model than for the reduced model ( 27 and 15\%); the relative decrease in reordering time was similar to the relative decrease in the number of non-zero elements ( 34 and 19\%). In contrast, for both models memory requirements for factoring and solution time were similar for the transformed and untransformed equations.

With SPARSPAK-A, similar results were also obtained for both models (i.e., little change in memory requirements but with reduction in solution time of 1.4 and $5 \%$ for transformed equations). Reordering times unexpectedly increased ( 42 and $110 \%$ ) for the transformed equations. The reason for this difference with SPARSPAK-A is the result of the different implementation of the minimum degree algorithm used in original SPARSPAK and SPARSPAK-A. The reordering algorithm used in SPARSPAK-A was 4 to 10 times more efficient, however, so this version of SPARSPAK may be preferable, depending on how many rounds of solutions are required. In conclusion, for the example data set and models used, transformation of the coefficient matrix resulted in a small improvement in reordering time with original SPARSPAK but resulted in a large increase in reordering time with SPARSPAK-A. The SPARSPAK-A version of SPARSPAK was much more efficient than the original version for reordering for both untransformed and transformed equations but was slightly slower in solution time than the original version. Other data sets and models may have different reordering and solution characteristics. 


\section{Implications}

The sequential transformation makes programming of multiple-trait mixed-model equations somewhat easier by forcing the residual variance-covariance matrix to be the identity matrix. Covariance components can be estimated for multiple traits by derivative-free restricted maximum likelihood from the transformed equations because the logarithm of the determinant of the coefficient matrix is an easily determined function of the logarithms of the determinant of the coefficient matrix after transformation and the transformation matrix. Some computations do not need to be repeated in each round of iteration with the transformed equations and memory requirements for storing the non-zero elements are less.

\section{Literature Cited}

Arnason, T. 1982. Prediction of breeding values for multiple traits in small non-random mating (horse) populations. Acta Agric. Scand. 32:171.

Boldman, K. G., and L. D. Van Vleck. 1991. Derivative-free restricted maximum likelihood estimation in animal models with a sparse matrix solver. J. Dairy Sci. 74:4337.

Chu, E., A. George, J. Liu, and E. Ng. 1984. SPARSPAK: Waterloo sparse matrix package user's guide for SPARSPAK-A. CS84-36, Dept. of Computer Sci., Univ. of Waterloo, ON, Canada.

Dempster, A. P., N. M. Laird, and D. B. Rubin. 1977. Maximum likelihood from incomplete data via the EM algorithm. J. R. Statist. Soc. Series B 39:1.

Garrick, D. J. 1988. Restricted maximum likelihood estimation of variance components for multiple traits with missing observations and an application to beef cattle. Ph.D. Thesis. Cornell Univ., Ithaca, NY.

George, A., J. Liu, and E. Ng. 1980. User guide for SPARSPAK: Waterloo sparse linear equations package. CS-78-30, Dept. of Computer Sci., Univ. of Waterloo, ON, Canada.

George, A., and E. Ng. 1984. A new release of SPARSPAK: The Waterloo sparse matrix package. Dept. of Computer Sci., Univ. of Waterloo, Waterloo, ON, Canada (Mimeo).

Graser, H.-U., S. P. Smith, and B. Tier. 1987. A derivative-free approach for estimating variance components in animal models by restricted maximum likelihood. J. Anim. Sci. 64:1362.

Gregory, K. E., L. V. Cundiff, and R. M. Koch. 1988. Germ plasm utilization in beef cattle. Beef Res. Progr. Rep. 3, ARS-71. USDA Meat Anim. Res. Ctr., Clay Center, NE.

Harville, D. A. 1977. Maximum likelihood approaches to variance component estimation and to related problems. J. Am. Stat. Assoc. 72:320.

Henderson, C. R. 1976. A simple method for computing the inverse of a numerator relationship matrix used in prediction of breeding values. Biometrics 32:69.

Henderson, C. R. 1977. Best linear unbiased prediction of breeding values not in the model for records. J. Dairy Sci. 60:783.
Henderson, C. R. 1984. Applications of linear models in animal breeding. University of Guelph Press, Guelph, ON, Canada.

Lawlor, T. J. 1984. Estimation of genetic and phenotypic parameters of milk, fat and protein yields of Holstein cattle under selection. Ph.D. Thesis. Cornell Univ,, Ithaca, NY.

Liu, J. 1983. On multiple elimination in the minimum degree algorithm. Tech. Rep. 83-03. Dept. of Computer Sci., York Univ., Toronto, ON, Canada.

Meyer, K. 1985. Maximum likelihood estimation of variance components for a multivariate mixed model with equal design matrices. Biometries 41:153.

Meyer, K. 1986. Restricted maximum likelihood to estimate genetic parameters-in practice. Proc. 3rd World Cong. Genet. Appl. Livest. Prod. 12:454. Lincoln, NE.

Meyer, K. 1988. DFREML-A set of programs to estimate variance components under an individual animal model. J. Dairy Sci. 71(Suppl. 2):33.

Meyer, K. 1989. Restricted maximum likelihood to estimate variance components for animal models with several random effects using a derivative-free algorithm. Genet. Sel. Evol. 21:317.

Meyer, K. 1991. Estimating variances and covariances for multivariate animal models by restricted maximum likelihood. Genet. Sel. Evol. 23:67.

Patterson, H. D., and R. Thompson. 1971. Recovery of inter-block information when block sizes are unequal. Biometrika 58:545.

Pollak, E. J., and R. L. Quaas. 1982. Alternative strategy for building multiple trait mixed model equations. J. Dairy Sci. 68(Suppl. 1): 102.

Quaas, R. L. 1976. Computing the diagonal elements and inverse of a large numerator relationship matrix. Biometrics 32:949.

Quaas, R. L. 1984. Linear prediction. In: BLUP School HandbookUse of Mixed Models for Prediction and for Estimation of (Co)Variance Components. p 158. Feb. 5-7, Animal Genetics and Breeding Unit, Univ. of New England, New South Wales, Australia.

Searle, S. R. 1979. Notes on variance component estimation: A detailed account of maximum likelihood and kindred methodology. Paper BU-673-M. Biometrics Unit, Cornell Univ., Ithaca, NY.

Smith, S. P., and H.-U. Graser. 1986. Estimating variance components in a class of mixed models by restricted maximum likelihood. J. Dairy Sci. 69:1165.

Sorensen, D. A., and B. W. Kennedy. 1983. The use of the relationship matrix to account for genetic drift in the analysis of genetic experiments. Theor. Appl. Genet. 66:217.

Sorensen, D. A., and B. W. Kennedy. 1984. Estimation of genetic variances from unselected and selected populations. J. Anim. Sci. 59:1213.

Sorensen, D. A., and B. W. Kennedy. 1986. Analysis of selection experiments using mixed model methodology. J. Anim. Sci. 63: 245.

Thompson, R. 1977. Estimation of quantitative genetic parameters. In: Proc. Int. Quant. Genetic Conf. pp 639-657. Ames, IA.

Walter, J. P., I. L. Mao, and B. D. Banks. 1986. An iterative algorithm for estimation of variance and covariance components in a multiple trait model using triangular decomposition of residual variance-covariance matrix and absorption of fixed effects. J. Dairy Sci. 69(Suppl. 2):207. 